\title{
Severe Falciparum Malaria in Iran:A Very Rare Case From an Endemic Region
}

\author{
Masood Ziaee ${ }^{1}$; Farshid Abedi ${ }^{2, *}$ \\ ${ }^{1}$ Hepatitis Research Center, Faculty of Medicine, Birjand University of Medical Sciences, Birjand, IR Iran \\ ${ }^{2}$ Faculty of Medicine, Mashhad University of Medical Sciences, Mashhad, IR Iran \\ *Corresponding author: Farshid Abedi, Faculty of Medicine, Mashhad University of Medical Sciences, Mashhad, IR Iran. Tel: +98-9155063062, Fax: +98-5118515001, E-mail: abedif@ \\ mums.ac.ir
}

Received: October 23, 2012; Revised: January 21, 2013; Accepted: February 23, 2013

\begin{abstract}
Introduction: Malaria is a protozoal disease, transmitted to humans by female Anopheles mosquito bite. Plasmodium falciparum, compared to other kinds of Plasmodium, causes more severe malaria and is associated with a higher mortality rate. Annually, one to three million deaths occur due to malaria, especially by P. falciparum.

Case Presentation: In this report, we introduce an Iranian patient suffering from P. falciparum. Peripheral blood smear for malaria parasites showed severe infection of $P$. falciparum, with 75 to 85 percent of red blood cells containing one to five parasites per cell. However, the patient revealed a fast response to treatment and a good prognosis, suggesting a high level of relative immunity in the patient. To confirm this hypothesis, we conducted a comparative study by comparing the rate of clinical response to treatment as well as the level of prognosis of our patient with similar patients from different regions around the world. These included some malaria cases (caused by $P$. falciparum) chosen from endemic and nonendemic regions, such as Africa, South Europe and Canada.

Discussion: The findings revealed that generally, patients from endemic regions significantly show a greater response to treatment and also a better prognosis in comparison to the patients from nonendemic regions. These differences can plausibly be attributed to a high level of relative immunity in endemic regions. Consequently, we would strongly support the hypothesis that response to treatment and prognosis of malaria is a matter of patients' living environment circumstances. In other words, people who live in endemic regions acquire a high relative immunity leading to a greater response to treatment and a better prognosis.
\end{abstract}

Keywords: Malaria; Falciparum; Immunity; Drug Therapy; Prognosis

\section{Introduction}

Malaria is a protozoan infection that is transmitted to humans through the bite of the Anopheles mosquito. This is the most important parasitic disease which is responsible for about one to three million deaths each year. Malaria today, as in the past, is a very big threat for tropical communities and nonendemic countries as well as travellers $(1,2)$. Annually, more than 2,700,000 people are at risk in malarious areas of Iran and about 3,000 malaria cases are reported (3). Annually, about 1,000 cases of malaria are reported in the United States, most of which are passengers or immigrants (4). Four species of Plasmodium including P. falciparum, P. malariae, $P$. ovale, and $P$. vivax, cause nearly all malaria infections in humans. However, almost all malaria-attributable mortalities are due to P. falciparum.

The human infection starts when the bite of an infected female Anopheles mosquito injects the Plasmodium sporozoites from the salivary gland into the human blood circulation. These microscopic malaria parasites rapidly travel through the bloodstream to the liver and begin the period of asexual reproduction. During this replication process, known as intrahepatic or preerythrocytic merogony step, 10,000 to more than 30,000 daughter merozoites are produced from sporozoites. Afterwards, the liver cell containing these merozoites ruptures and releases them into the bloodstream. At this stage, the infection is symptomatic. The merozoites, immediately after entering the bloodstream, invade the red blood cells (RBCs) and become trophozoite. Since P. vivax, P. ovale, and P. malariae have tendency to invade mature RBCs, parasitemia rarely exceeds $2 \%$; whereas in Falciparum malaria, severe parasitemia occurs due to invasion to RBCs of all ages (2). In this paper we introduce an Iranian case of severe malaria rapidly responding to treatment with intravenous quinine sulfate compared to other cases of severe malaria in the world.

\section{Case Presentation}

In January 2001, a 32-year-old man referred to Montaserieh hospital of Mashhad, Iran, with a history of fevers and vomiting 10 days after returning from Pakistan. He also complained of dark urine and hematuria. He had no history of receiving prophylactic antimalarial drugs.

Implication for health policy/practice/research/medical education:

In this article, we introduce an Iranian patient suffering from $P$. falciparum. Peripheral blood smear for malaria parasites showed a severe infection of $P$. falciparum. The findings revealed that generally, patients from endemic regions significantly show a greater response to treatment and also a better prognosis in comparison to the patients from non-endemic regions.

Copyright (c) 2014, Ahvaz Jundishapur University of Medical Sciences; Published by Kowsar Corp. This is an open-access article distributed under the terms of the Creative Commons Attribution License, which permits unrestricted use, distribution, and reproduction in any medium, provided the original work is properly cited. 
He had previously been hospitalized in a small hospital with a diagnosis $P$. vivax infection and had received oral chloroquine therapy. Then the patient was referred to a specialized hospital in Mashhad city due to worsening symptoms. At admission, he had a temperature of $38.5^{\circ} \mathrm{C}$ and his other vital signs and consciousness level were normal. On physical examination, the patient had a tender abdomen and his spleen was palpable $5 \mathrm{~cm}$ below the left costal margin.

In laboratory studies, hemoglobin was $11.5 \mathrm{~g} / \mathrm{dL}$, hema- tocrit $34 \%$, white blood cell count $16,000 / \mathrm{mm}^{3}$, and platelet count was $45,000 / \mathrm{mm}^{3}$. His creatinine level was 1.9 $\mathrm{mg} / \mathrm{dL}$. Serum was assayed for total bilirubin (Bil), lactate dehydrogenase (LDH), aspartate aminotransferase (AST), and alanine aminotransferase (ALT), using commercially available kits. The serum level of Bil was $2.3 \mathrm{mg} / \mathrm{dL}$, LDH $1680 \mathrm{U} / \mathrm{L}$ (normal level: 80 -100 U/L), AST $106 \mathrm{U} / \mathrm{L}$, and ALT 29 U/L. Peripheral blood smear examination showed severe infection with P. falciparum and 75 to $85 \%$ of RBCs contained one to five parasites per cell (Figure 1).

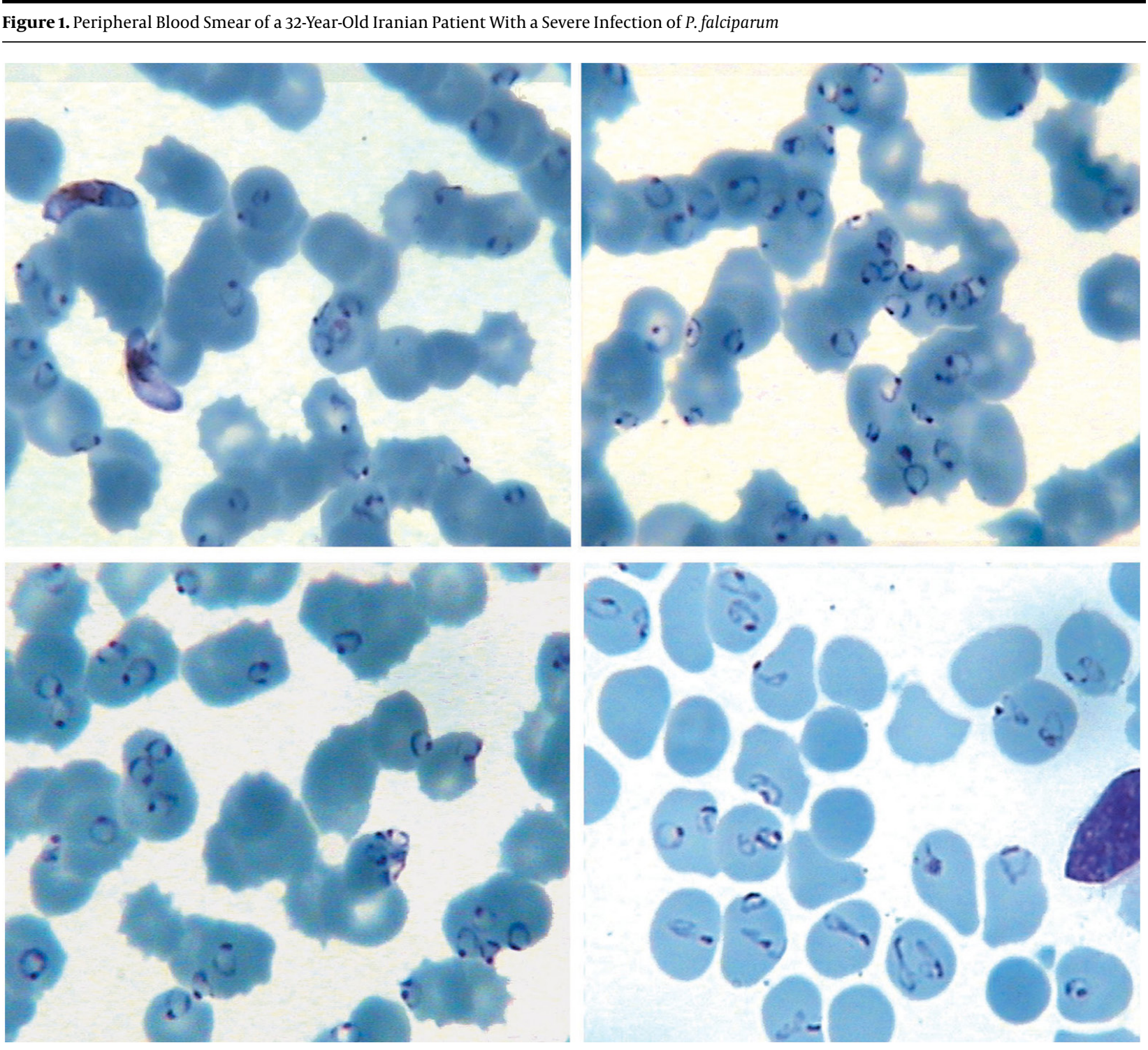

Among RBCs, 75 to $85 \%$ contained one to five parasites per cell (Giemsa stain, X1000)

Patient probably had a mixed infection with P. vivax and $P$. falciparum that the $P$. vivax infection had been treated with oral chloroquine, but chloroquine-resistant $P$. falciparum remained and parasitemia had increased.

The patient was treated with intravenous quinine sul- fate and oral primaquine. Then, he received primaquine (30 $\mathrm{mg}$ a day for 14 days) after the symptoms improvement for treating the probable mixed malaria infection. The improvement was considerable, so that parasitemia decreased by $40 \%$ after 24 hours and $1 \%$ after 4 days of 
posttreatment. During the treatment process, the patient developed dark urine and elevated creatine phosphokinase; thus, progression to acute renal failure was considered for him. Acute respiratory distress syndrome (ARDS) was exhibited in bilateral pulmonary infiltrates on the chest X-ray; also, he presented confusion due to cerebral malaria or superimposed a Gram-negative infection, so ceftriaxone was prescribed. However, patient was discharged after 10 days with no requirement for intubation, hemodialysis, or ICU admission. He had a good general condition at the last follow-up visit.

\section{Discussion}

Severe falciparum malaria is a medical emergency that requires intensive care. Parenteral quinine or quinidine should be administered if any doubt exists about drug resistance. In very ill patients, blood exchange transfusion should be considered and it is indicated in high parasitemia (more than 30\%) (5). The patient presented in this paper was important in two aspects: one was because of high parasitemia which was certainly a very rare condi- tion and the only similar reported case in the world with high parasitemia as our patients was a Swiss case (with a parasitemia of $70-80 \%$ ), and second, the rapid response to treatment with intravenous quinine without the necessity of blood exchange or other supportive care proceedings (such as hemodialysis, ventilator, or ICU admission) and improvement after 10 days hospitalization.

Outcomes of sever malaria differ in immune and nonimmune patients (6). We found some reports of immune and nonimmune patients with severe malaria. The first case was a 34-year-old Swiss patient with a history of travel to Madagascar presenting severe P. falciparum malaria in which $70-80 \%$ of RBCs in the peripheral blood smear were infected (Figure 2) (7). The treatment course took about 3 months and he was hospitalized during this process. In addition to administration of intravenous quinine sulfate, he underwent blood exchange transfusion as well as laparotomy and splenectomy due to splenic rupture. Also, he was placed on mechanical ventilation, and underwent hemodialysis. Although the rate of parasitemia was similar to that of our patient, response to the treatment was completely different (7).

Figure 2. Peripheral Blood Smear in a 34-Year-old Swiss Patients With Severe Malaria
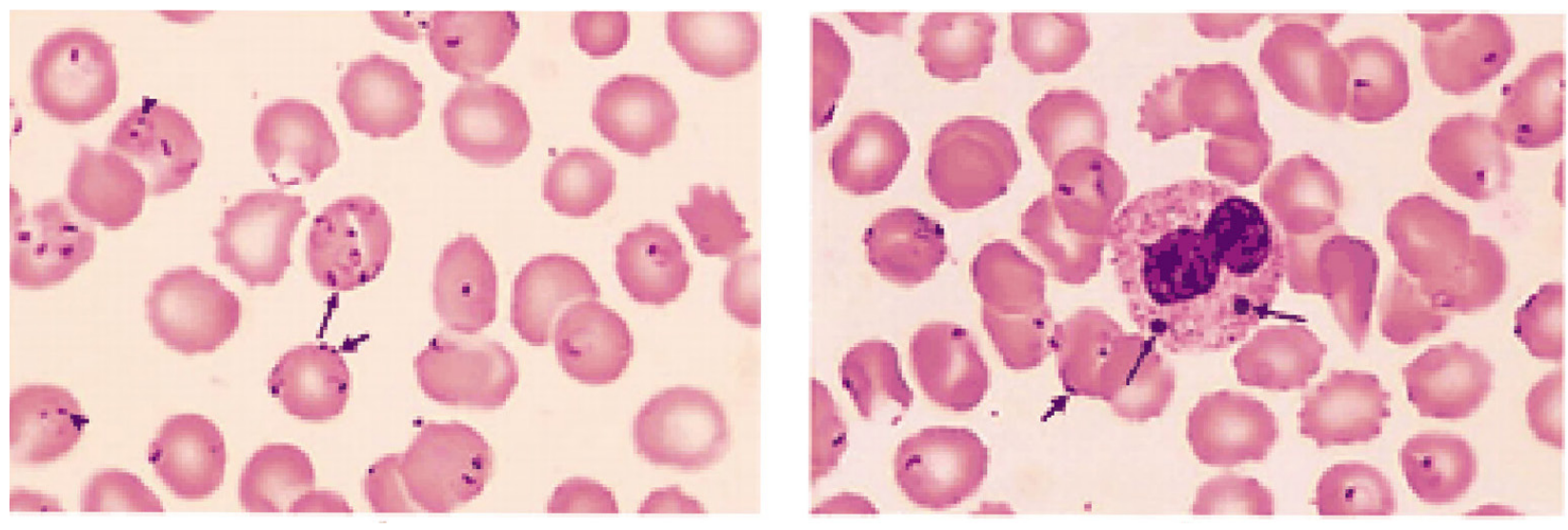

Among RBCs, 70 to $80 \%$ contained up to five $P$. falciparum cells (7).

The other case was a 54-year-old patient, progressed to severe malaria ( $P$. falciparum) after returning from Kenya with a parasitemia rate of $10.4 \%$ (8). Treatment with intravenous quinine and doxycycline were initiated for him. Despite the elimination of parasites from the blood, patient died from pulmonary edema and heart failure due to water and electrolyte abnormalities. Although the percentage of parasitemia was lower and even the treatment contained broad-spectrum drugs, he was a nonimmune case from a nonendemic region and so had a poorer prognosis than the two previously mentioned cases.

The other patient was a 17-year-old UK-resident woman who was born in Nigeria. She presented a maximum parasitemia of 30\% (P. falciparum) after returning from her native country. Although she was admitted to ICU and undergone ventilation and hemodialysis, she was discharged with good general conditions and no need for blood exchange transfusion on the day 10 after the admission. In this case, although the disease progress was associated with more complications than ours, no need for blood exchange as well as the short length of hospital stay (10 days) indicated the relative immunity of patient which was due to her birthplace and history of living in an endemic region (9).

Other case was a 29-year-old Canadian man who had non-specific symptoms of malaria after returning from Zambia. The peripheral blood smear was negative for malarial parasite. The patient's consciousness was impaired 48 hours later and he died in the next few hours. P. falciparum was reported as the cause of death in autopsy. 
Although this patient received no medical treatment, the rapid and fatal course of malaria infection even with negative parasitemia in a nonimmune patient living in a nonendemic region, draws our attention to the importance of the relative immunity of people living in endemic regions (10).

Finally, in another article, four patients with P. falciparum were introduced (two 50-year-old Canadian patients, male and female, with parasitemia of $1 \%$, a 28 -yearold Norwegian patient with parasitemia of $2 \%$, and a 64-year-old Indian patient with parasitemia of $6 \%$ ). The Indian patient, compared to the three others (who lived in nonendemic regions) despite a higher rate of parasitemia, had lower symptoms and a faster response to treatment (11). Presumably, the theory of a relative immunity existence among people living in the malaria endemic regions, leads to a faster response to treatments and better prognosis. This is quite reasonable considering the collected data and cases from around the world. Therefore, the indications of blood exchange, ICU admission, laparotomy, and splenectomy, as well as the disease prognosis in severe malaria, can be different based on a person's location and immunity.

\section{Acknowledgements}

We wish to thank staffs of Department of Infectious Disease in Mashhad University of Medical Sciences.

\section{Authors' Contribution}

Masood Ziaee, first edition and drafting of the manuscript. Farshid Abedi, corresponding author and contribution to abstracting the data and preparing the manuscript.

\section{Financial Disclosure}

Authors have no financial interests related to the manuscript.

\section{Funding/Support}

The authors have nothing to disclose.

\section{References}

1. Setiawan B. Ministry of Health Republic of Indonesia. Current malaria management: guideline 2009. Acta Med Indones. 2010;42(4):258-61.

2. Trampuz A, Jereb M, Muzlovic I, Prabhu RM. Clinical review: Severe malaria. Crit Care. 2003;7(4):315-23.

3. Hanafi-Bojd AA, Vatandoost H, Oshaghi MA, Haghdoost AA, Shahi $M$, Sedaghat MM, et al. Entomological and epidemiological attributes for malaria transmission and implementation of vector control in southern Iran. Acta Trop. 2012;121(2):85-92.

4. Vicas AE, Albrecht H, Lennox JL, del Rio C. Imported malaria at an inner-city hospital in the United States. Am J Med Sci. 2005;329(1):6-12.

5. Pasvol G. The treatment of complicated and severe malaria. $\mathrm{Br}$ Med Bull. 2005;75-76:29-47.

6. Checkley AM, Smith A, Smith V, Blaze M, Bradley D, Chiodini PL, et al. Risk factors for mortality from imported falciparum malaria in the United Kingdom over 20 years: an observational study. BMJ. 2012;344.

7. Chalandon Y, Kocher A. Images in clinical medicine. Severe malaria. N Engl J Med. 2000;342(23):1715.

8. von Mach MA, Hansen T, Ehrenthal W, Sauer O, Weilemann LS. [Fulminant pulmonary edema in falciparum malaria [corrected]l. Dtsch Med Wochenschr. 2003;128(16):874-6.

9. Moore DA, Jennings RM, Doherty TF, Lockwood DN, Chiodini PL, Wright SG, et al. Assessing the severity of malaria. BMJ. 2003;326(7393):808-9.

10. Kain KC, MacPherson DW, Kelton T, Keystone JS, Mendelson J, MacLean JD. Malaria deaths in visitors to Canada and in Canadian travellers: a case series. CMAJ. 2001;164(5):654-9.

11. Chung SY, Das R, Oon CT. Treatment of severe falciparum malaria with artesunate and mefloquine. Singapore Med J.1998;39(5):20810. 